\title{
Interface roughness effects on coercivity and exchange bias
}

Ana L. Dantas, G. O. G. Rebouças, A. S. W. T. Silva, and A. S. Carriço

Citation: Journal of Applied Physics 97, 10K105 (2005);

View online: https://doi.org/10.1063/1.1847931

View Table of Contents: http://aip.scitation.org/toc/jap/97/10

Published by the American Institute of Physics

\section{Articles you may be interested in}

Influence of interface roughness on the exchange bias of $\mathrm{Co} / \mathrm{CoO}$ multilayers

Journal of Applied Physics 113, 17D707 (2013); 10.1063/1.4795437

Antiferromagnetic iridium-manganese intermediate layers for perpendicular recording media (invited) Journal of Applied Physics 105, $07 B 738$ (2009); 10.1063/1.3080886

Thermal hysteresis of interface biased ferromagnetic dots Journal of Applied Physics 102, 123907 (2007); 10.1063/1.2827478

Kerr observations of asymmetric magnetization reversal processes in CoFe/lrMn bilayer systems Journal of Applied Physics 93, 5491 (2003); 10.1063/1.1562732

Unidirectional coercivity enhancement in exchange-biased $\mathrm{Co} / \mathrm{CoO}$ Applied Physics Letters 81, 1270 (2002); 10.1063/1.1498505

High coercivity and exchange coupling of $\mathrm{Ni} / \mathrm{NiO}$ nanocomposite film Journal of Applied Physics 97, 10 K306 (2005); 10.1063/1.1851424

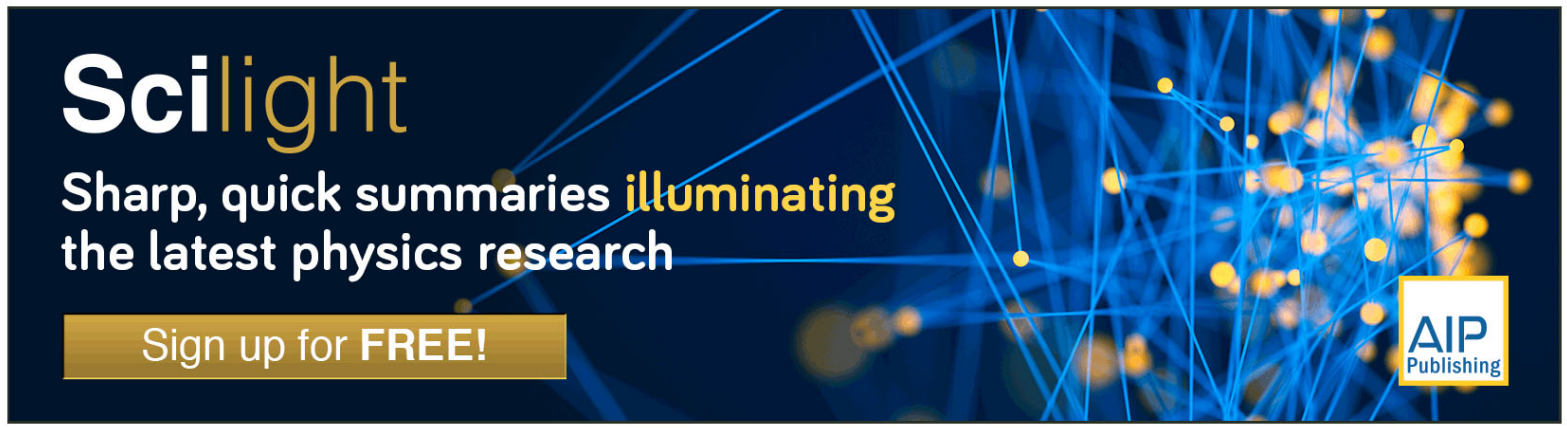




\title{
Interface roughness effects on coercivity and exchange bias
}

\author{
Ana L. Dantas ${ }^{a)}$ \\ Departamento de Física, Universidade do Estado do Rio Grande do Norte, \\ Mossoró-Rio Grande do Norte 59610-210, Brasil

\begin{abstract}
G. O. G. Rebouças, A. S. W. T. Silva, and A. S. Carriçob)
Departamento de Física Teórica e Experimental, Universidade Federal do Rio Grande do Norte, Natal - Rio Grande do Norte 59078-970, Brasil
\end{abstract}

(Presented on 9 November 2004; published online 13 May 2005)

\begin{abstract}
We report model calculations of the hysteresis loops of exchange-coupled ferromagnet/ antiferromagnet bilayers with monolayer-scale roughness and show that the loops are affected by the combined effect of the interface field strength, the degree of magnetic roughness and magnetostatic effects. The magnetization reversal may occur via domain-wall nucleation at the edges of monoatomic interface steps or coherent magnetization rotation. A magnetic phase diagram is constructed for a 10-nm-thick Fe film, subjected to nanometer-scale interface roughness. (C) 2005 American Institute of Physics. [DOI: 10.1063/1.1847931]
\end{abstract}

The influence of defects on the form of hysteresis loops and coercivity is an issue of recurring interest. In bulk samples the reduction of coercive field, compared with the prediction of the Stonner-Wolfarth coherent rotation (CR), has been explained by invoking the role of lattice defects, where the magnetic parameters may vary considerably, ${ }^{1}$ which act as domain-wall nucleation centers. The surface or interface spins of thin-film magnetic multilayers may represent a considerable fraction of the system. Therefore, the variations in the magnetic parameters for the surface or interface spins, due to monolayer roughness, may affect the equilibrium configuration of the whole system of spins. For instance, local variations in the magnetic coordination of the surface spins produces relevant changes in the hysteresis of ultrathin films. ${ }^{2}$

The magnetic properties of exchange-coupled ferromagnetic/antiferromagnetic (F/AF) bilayers are the focus of a great deal of research effort, motivated by fundamental questions, related to how the atomic scale interface structure may control the bilayer macroscopic properties, and the promising potential for the development of magnetoelectronic devices. ${ }^{3}$ The exchange bias, consisting of a shift of the hysteresis loop, and the coercivity are both affected by the interface microscopic structure.

Monolayer interface roughness in $(\mathrm{F} / \mathrm{AF})$ bilayers may lead to various magnetic phases. A correlation between the domains in the F film and the direction of the AF interface spins is expected if magnetic coherence is preserved in large interface areas, as reported in a study of a $\mathrm{LaFeO}_{3} / \mathrm{Co}$ bilayer, using magnetic circular dichroism. ${ }^{4}$

In this paper we study the hysteresis loops of exchangecoupled ferromagnet/antiferromagnet bilayers with monolayer-scale roughness and discuss the impact of the magnetostatic interaction on the coercivity and exchange bias field. We use a simplified model of interface roughness,

\footnotetext{
${ }^{a)}$ Electronic mail: ana@uern.br

${ }^{b)}$ Electronic mail: acarrico@dfte.ufrn.br
}

as depicted in Fig. 1, in which the AF interface plane has a periodic distribution of islands, where the AF spins have the opposite direction of the background. This corresponds to a two-sublattice noncompensated AF plane [such as the surface of a (100) $\mathrm{FeF}_{2}$ film], with monolayer-height islands containing spins of one sublattice on a surface containing spins of the opposite sublattice.

We consider the AF spins to be frozen in the AF configuration and study the F film magnetization reversal. We apply the results for a thin Fe film, and the magnetic state of the film is represented by a monolayer of cubic cells of 10 $\mathrm{nm}$ in size, with the energy including the exchange coupling between neighboring cells, the anisotropy energy, the interface coupling to the AF spins, and the external and dipolar fields. The energy is given by

$$
\begin{aligned}
E= & \sum_{i, j} \sum_{m, n}-J \overrightarrow{\boldsymbol{S}}_{i, j} \overrightarrow{\boldsymbol{S}}_{m, n} \\
& -\sum_{i, j}\left[\frac{K}{2}\left(S_{i, j}^{z}\right)^{2}+g \mu_{B} \overrightarrow{\boldsymbol{S}}_{i, j} \cdot\left(\overrightarrow{\boldsymbol{H}}_{\mathrm{INT}}+\overrightarrow{\boldsymbol{H}}+\overrightarrow{\boldsymbol{H}}_{D_{i, j}}\right)\right] .
\end{aligned}
$$

The cell size was chosen smaller than the Fe exchange length, and the magnetic parameters ${ }^{5}$ have been renormalized to take into account the cell size in the effective param-

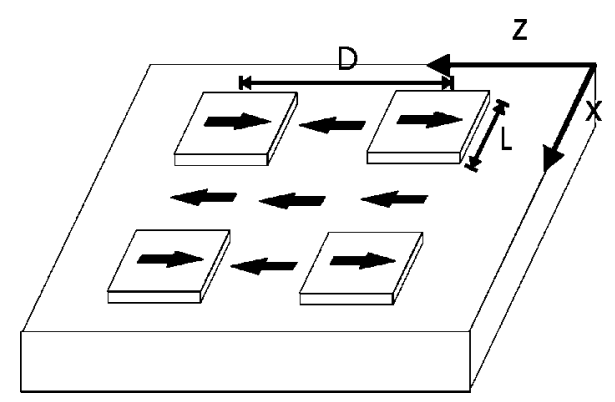

FIG. 1. Schematic view of the AF interface plane showing island of sublattice 2 on a background of sublattice 1. Four magnetic unit cells of periodic structure of the interface plane are shown and the dimensions, $D$ and $L$, are defined in the text. 


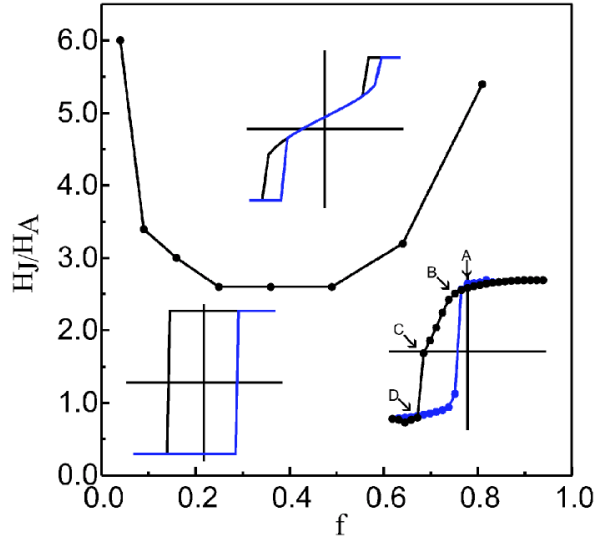

FIG. 2. Phase diagram for $D=20$. The line separates the regime of uniform rotation with the square hysteresis loops, from that of reversal nucleated at domain walls on the island edges, with double loop hysteresis.

eters used in Eq. (1). The dipolar field $\left(H_{D}\right)$ at each cell $(i, j)$ has been summed up to terms of the order of $1 \%$. Periodic boundary conditions were used for a magnetic unit cell containing $D$ cubic cells and the scale of interface roughness is studied using the fraction $f=L^{2} / D^{2}$ of ferromagnetic spins exchange coupled to the AF spins in the islands. We consider interface antiferromagnetic coupling, and the interface field $\left(\overrightarrow{\boldsymbol{H}}_{\mathrm{INT}}\right)$ is parallel to the uniaxial anisotropy axis ( $\hat{z}$ axis) and has opposite directions for the $\mathrm{F}$ spins over the islands $\left(\overrightarrow{\boldsymbol{H}}_{\mathrm{INT}}=-H_{J} \hat{z}\right)$ or the channels between the islands $\left(\overrightarrow{\boldsymbol{H}}_{\mathrm{INT}}\right.$ $\left.=H_{J} \hat{z}\right)$. Throughout the text we use the fields in units of the anisotropy field $\left[H_{A}=K /\left(g \mu_{B} S\right) \cong 0.5 \mathrm{kOe}\right]$.

The hysteresis loops were calculated using a selfconsistent local-field method ${ }^{6}$ starting with large positive or negative values of the external field, and for at each new field value the equilibrium configuration corresponding to the previous value of the external field has been used to initialize the magnetic configuration. The spin variables $\left(S_{i, j}\right)$ represent the magnetic moment per cubic cell and are allowed to relax to the local field, with the restriction of no component perpendicular to the $\mathrm{F}$ film surface ( $x z$ plane).

Without dipolar effects the shape of the hysteresis loops depends on the interface field strength and on the size of the areas of magnetic coherence of the AF interface plane. The magnetic phases depend on an energy balance that involves the gain of interface energy, controlled by the strength of the interface exchange field and the areas of magnetic coherence in the AF interface plane, and the energy cost of forming domain walls, which is proportional to the perimeter of the coherent areas of the AF interface plane. In Fig. 2 we show the magnetic phase diagram, classifying the hysteresis loops according to the values of the interface field strength $\left(H_{J}\right)$ and the value of $f$. We have chosen $D=20$ which corresponds to a magnetic cell of $200 \times 200 \mathrm{~nm}^{2}$ area. For large values of the interface field strength we have found hysteresis curves with double loops, as shown in the inset on the upper part of Fig. 2. The loops are shifted towards the direction opposite to the average interface field, and the reversal occurs via domain-wall nucleation at the island edges. For small values of $f$, or $f \cong 1$, there is no room for domain walls to form,

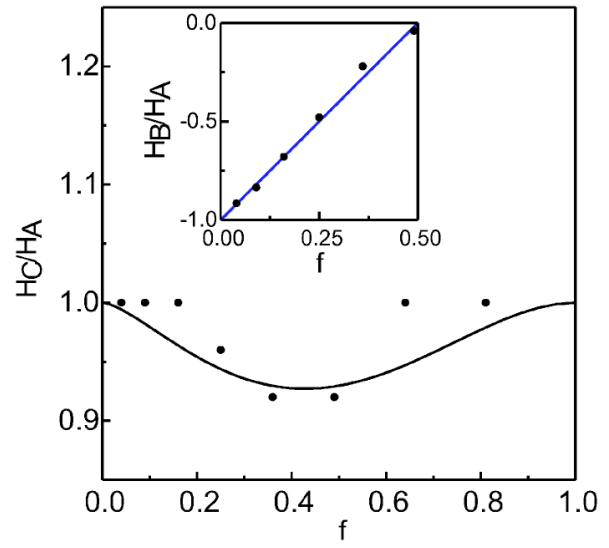

FIG. 3. Coercivity as a function of $f$ for $D=20$ and $H_{J}=H_{A}$.

either in the channels between islands or inside the islands, and large values of the interface field strength are required to nucleate domain walls.

If the interface field strength is of the order of the anisotropy field, we have found CR magnetization reversal, with a loop shift corresponding to the average interface field. In Fig. 3 we show that there is a small reduction of coercivity. On both branches of the hysteresis loop, there is a fraction of the spins (either $f$ or $1-f$ ), for which the interface field favors the magnetization reversal. Spins on opposite sides of the island edges are exchange coupled, thus the whole set of spins may rotate together, reducing the strength of the external field required for the magnetization reversal. The reduction is larger for $f \cong 0.5$, as shown in Fig. 3 , and is negligible if either $f \cong 0$ or $f \cong 1$. In these limits the number of spins that might nucleate reversal is too small. The continuous curve in Fig. 3 is obtained from an analytical model for the instability of the remanent state in both branches of the hysteresis. ${ }^{7}$ The effective exchange bias field differs very little from the average value of the interface field $\left[\left\langle H_{\mathrm{INT}}\right\rangle\right.$ $\left.=(1-2 f) H_{J}\right]$, as shown in the inset of Fig. 3 .

The dipolar field is one order of magnitude larger than the anisotropy field. Thus, one finds interesting dipolar effects if the interface field is of the same order of the anisotropy field. For $H_{J}=H_{A}$ the hysteresis loop resembles a CR loop, as shown in the inset (bottom right) of Fig. 2. We selected a nearly compensated interface, with $D=20$ and $L / D=0.7$, which is close to having $f=0.5$. In the absence of magnetostatic effects the loop shift is rather small. There are 196 cells over the islands and 204 cells in the channels between islands. Thus the average interface field is $\left\langle H_{\mathrm{INT}}\right\rangle$ $=8 H_{J} / 400$, which is a small fraction of the anisotropy field. The exchange bias field is $H_{b}=-0.02 H_{A}$.

The magnetostatic effects lead to a reduction of coercivity and an enhancement of the bias field. Spin configurations for selected points in the upper branch of the loop (points $A, B, C$, and $D$, shown in the inset of Fig. 2) are shown in Fig. 4. Note that the two edges of the islands are not equivalent. At the edge running parallel to the anisotropy field, the transition from the profile at the islands to that outside is similar to that found in a Nèel wall. Whereas at the edges perpendicular to the $\mathrm{F}$ easy axis, a domain wall conforming with the trends imposed by the interface field would have a 

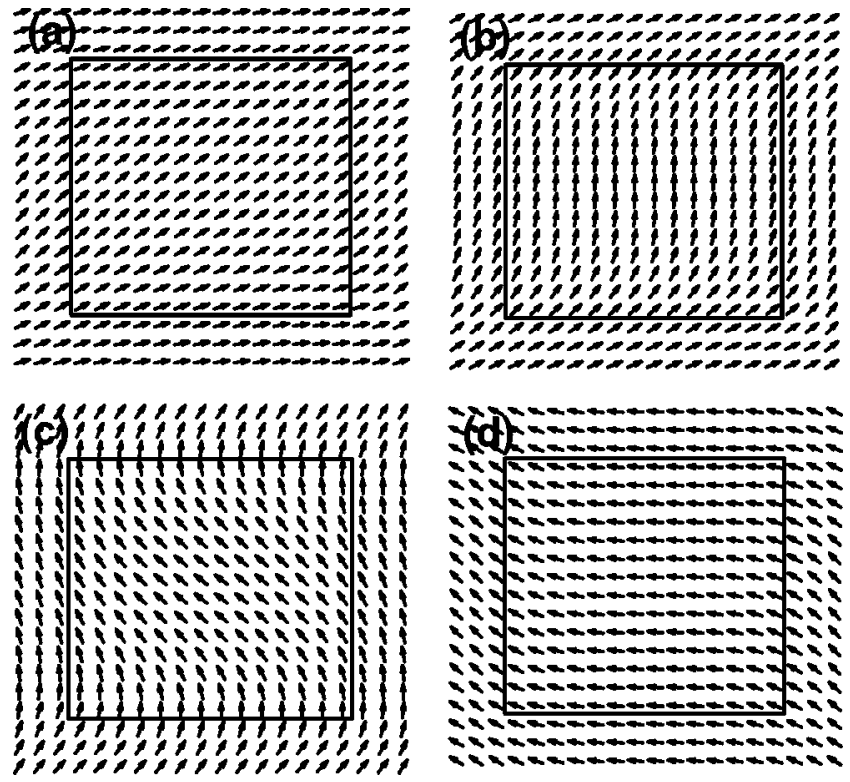

FIG. 4. Spin configurations at selected points of the upper branch of the hysteresis loop shown in the bottom-right inset of Fig. 2. We use $D=20$ and $L / D=0.7$, which correspond to a nearly compensated interface $(f \approx 0.5)$. The values of the external field are (a) $H=0$, (b) $H=-0.32 H_{A}$, (c) $H=-1.28 H_{A}$, and (d) $H=-1.44 H_{A}$, as marked (points $A, B, C$, and $D$ ) in the hysteresis curve shown in Fig. 2

built-in charge. This difference between the edges is visible in the spin configurations that form during the reversal.

The reversal nucleates at the spin over the islands. The dipolar field of the spins in the channels enforces the effect of the interface field $\left(-H_{J} \hat{z}\right)$ on the spins over the islands, reducing of the value of the external field strength required for the magnetization reversal. The combined effect of the interface and dipolar field leads to a nonuniform gradual rotation of the spins over the islands, which dragg the spins over the channels. For a small value of the external field strength $\left(H=-0.32 H_{A}\right)$ the island spins are in a perpendicular state which evolves to saturation in the $-\hat{z}$-axis direction passing through $S$-like states, as shown for $H=-1.28 H_{A}$ in Fig. 4(c). Similar configurations are found in the lower branch of the loop (not shown), leading to a relevant reduction of the coercivity and a bias field of $H_{b} \cong-0.8 H_{A}$. This enhancement of the bias field, by almost one order of magnitude, results from the fact that the dipolar field is one order of magnitude larger than the interface field.

Coercivity enhancement is commonly found in F/AF bilayers and has been attributed to grains either in the $\mathrm{F}$ or $\mathrm{AF}$ material, and the modifications in the AF order of the substrate, including spin-flop-like coupling. ${ }^{8,9}$ Our choice of large anisotropy AF substrate for the present study allows a simpler discussion of the magnetostatic effects. We suggest that the magnetostatic interaction lowers the threshold value of $H_{J}$ for CR, producing a reduction in the coercivity and an enhancement of the exchange bias. We are currently investigating this effect in vicinal F/AF bilayers. In these systems the dipolar effects may be controlled by choosing the orientation of the miscut angle with respect to the anisotropy field of the F film. ${ }^{9}$

\section{ACKNOWLEDGMENTS}

This work was partially financed by the Brazilian research agencies CNPq, CAPES, and FAPERN/RN.

\footnotetext{
${ }^{1}$ See, for instance, H. Zijlstra, in Ferromagnetic Materials, edited by E. P. Wohlfarth (North-Holland, Amsterdam, 1982), Vol. 3.

${ }^{2}$ E. J. Escorcia-Aparicio, J. H. Wolf, H. J. Choi, W. L. Ling, R. K. Kawakami, and Z. Q. Qiu, Phys. Rev. B 59, 11892 (1999); R. A. Hyman, A. Zangwill, and M. D. Stiles, ibid. 60, 14830 (1999).

${ }^{3}$ J. Nogués and I. K. Schuller, J. Magn. Magn. Mater. 192, 203 (1999); M. D. Stiles and R. D. McMichael, Phys. Rev. B 59, 3722 (1999); B. Dieny, V. S. Speriosu, S. S. P. Parkin, B. A. Gurney, D. R. Wihoit, and D. Mauri, ibid. 43, 1297 (1991); H. D. Chopra, D. X. Yang, P. J. Chen, D. C. Parks, and W. F. Egelhoff Jr., ibid. 61, 9642 (2000); A. M. Zhang et al., J. Appl. Phys. 95, 7294 (2004).

${ }^{4} \mathrm{~F}$. Nolting et al., Nature (London) 405, 767 (2000).

${ }^{5}$ O. Fruchart, J. P. Nozières, W. Wernsdorfer, D. Givord, F. Rousseaux, and D. Decanini, Phys. Rev. Lett. 82, 1305 (1999).

${ }^{6}$ A. L. Dantas and A. S. Carriço, Phys. Rev. B 59, 1223 (1999).

${ }^{7}$ For CR reversal we use $\theta_{-}$and $\theta_{+}$to describe the magnetic state of the spins over the islands and over the channels between islands. The reversal fields are calculated from the roots of the eigenvalues of the stability matrix, constructed from the second derivatives of the magnetic energy with respect to $\theta_{-}$and $\theta_{+}$.

${ }^{8}$ L. Wang et al., Phys. Rev. B 66, 184411 (2002); J. Nogués et al., ibid. 61, R6455 (2000); T. C. Schulthess and W. H. Butler, Phys. Rev. Lett. 81, 4516 (1998); T. J. Moran and I. K. Schuller, J. Appl. Phys. 79, 5109 (1996).

${ }^{9}$ A. L. Dantas, G. O. G. Rebouças, A. S. W. T. Silva, and A. S. Carriço
} (unpublished). 